\title{
Single Dose Therapy of Zoledronic Acid for the Treatment of Transient Osteoporosis of Hip
}

\author{
Sanjay Agarwala, MD, Mayank Vijayvargiya, MD
}

\author{
Department of Orthopedics, P.D. Hinduja National Hospital and Medical Research Centre, Mumbai, India
}

Objective To shorten the natural course of transient osteoporosis of hip (TOH), which is a self-limiting idiopathic condition and requires 6-12 months for the resolution of symptoms, various therapies including treatment with bisphosphonates have been attempted. There exist fewer case reports evaluating the effect of bisphosphonates in $\mathrm{TOH}$ and most of them lack radiographic evidence of resolution of disease. The present study was carried out with an aim to evaluate the clinical and radiological outcomes of TOH patients subsequent to treatment with a single dose of zoledronic acid.

Methods Data of 19 adult male and non-pregnant female patients with TOH treated with zoledronic acid were included in the study. Efficacy was assessed using changes in clinical signs and symptoms, visual analogue scale pain score, and changes in bone marrow edema (BME) in magnetic resonance imaging (MRI).

Results Mean age of the patients was 42.1 years, 17 being males. The mean time of onset of symptom was 4 weeks. Subsequent to treatment, the patients were pain-free and had no limp within an average of 2.8 weeks (range, 2-5 weeks) and remained asymptomatic at a mean follow-up of 35 months (range, 6-54 months). The post-treatment MRI showed resolution of BME in approximately 84\% (16/19) of patients at 3 months. No major adverse events were reported. None of the patients progressed to avascular necrosis at their last follow-up.

Conclusion Intravenous single dose administration of zoledronic acid provides early pain relief and complete reversal of TOH. Consequently, zoledronic acid is proposed as a new paradigm in the management of TOH.

Keywords Transient osteoporosis of hip, Osteonecrosis, Bisphosphonates, Diphosphonates, Visual analog scale, Magnetic resonance imaging

\section{INTRODUCTION}

Transient osteoporosis of the hip (TOH), also known as transient bone marrow edema (BME) syndrome, is a rare, self-limiting condition of unknown etiology usually affecting middle-aged males, but also known to occur in females during the third trimester of pregnancy. TOH is characterized by painful limp and osteopenia of the affected joint [1]. In the early stage, the signs, symptoms, and the radiographic appearance are quite similar to

Received December 1, 2018; Accepted January 4, 2019

Corresponding author: Sanjay Agarwala

Department of Orthopedics, P.D. Hinduja National Hospital and Medical Research Centre, Veer Savarkar Marg, Mahim, Mumbai 400016, India. Tel: +91-986-944-6644, Fax: +91-22-2444-0425, E-mail: drsa2011@gmail.com

ORCID: Sanjay Agarwala (http://orcid.org/0000-0002-3146-2019); Mayank Vijayvargiya (https://orcid.org/0000-0002-2405-0411).

(c) This is an open-access article distributed under the terms of the Creative Commons Attribution Non-Commercial License (http://creativecommons.org/ licenses/by-nc/4.0) which permits unrestricted noncommercial use, distribution, and reproduction in any medium, provided the original work is properly cited. Copyright $\odot 2019$ by Korean Academy of Rehabilitation Medicine 
avascular necrosis (AVN) and only magnetic resonance imaging (MRI) can provide an exact diagnosis of TOH [2]. This is a rare condition and there exist fewer case reports published in the world literature.

Studies have demonstrated $\mathrm{TOH}$ as a self-limiting condition with spontaneous recovery; however, it may take up to 12-24 months for complete resolution of symptoms. Therefore, to shorten the natural history and attain early resolution, numerous pharmacological therapies have been attempted including treatment with bisphosphonates, calcitonin, and prostacyclin [3-5]. Whilst other therapies have shown inconsistent results, treatment with bisphosphonates have shown the promising result in shortening the clinical course of the disease $[3,6,7]$.

Studies evaluating the role of alendronate have shown that it may take 4 to 17 weeks for complete resolution of the symptoms $[3,6,8]$. On the contrary, it has been reported that intravenous bisphosphonates provide early pain relief and resolution of symptoms as compared to alendronate [8-10]. This finding can be substantiated based on the stated findings that zoledronic acid with its higher bioavailability and faster onset of action can provide early relief from symptoms. Studies have shown that the suppression of bone resorption occurs within approximately 3 months of initiation of oral bisphosphonate therapy (alendronate) regardless of dosing frequency, but is more rapid after intravenous zoledronic acid administration [11]. Also, a biomarker evaluation reported by Saag et al. [12] stated detectable levels of N-telopeptide of type I collagen (NTX) in urine at 1 week after zoledronic acid administration, and 12 weeks in case of alendronate.

There exist very few studies, mostly case reports, assessing the effects of zoledronic acid in $\mathrm{TOH}$ [8]. However, case series documenting the beneficial role of zoledronic acid is lacking. Further, the reported studies have not documented the radiographic improvement in terms of resolution of the disease process in MRI. We conducted this study to evaluate the clinicoradiological outcome of management of TOH with zoledronic acid administration.

\section{MATERIALS AND METHODS}

The present study involved retrospective analyses of data extracted from the medical records of 19 patients with TOH. The study was reviewed and approved by the Institutional Review Board of P.D. Hinduja National Hospital \& Medical Research Centre (No. 964-15-SAg). Informed written consent was obtained from all the patients before starting the treatment. The patients visited the orthopedic department of a tertiary care center in Mumbai between June 2010 and December 2015. Adult males and non-pregnant females with $\mathrm{TOH}$ who were treated with zoledronic acid and with at least 12 weeks follow-up data were included in the study. Medical records including X-rays and MRIs (before and after the therapy) of the patients were critically evaluated for the availability of data before inclusion. The institution followed a standard clinical algorithm for diagnosis and treatment of $\mathrm{TOH}$.

The study patients were diagnosed with TOH based on history, clinical examination, and radiological evaluation. Presence of BME on MRI confirmed the diagnosis of TOH along with the absence of focal defects and subchondral changes, which ruled out AVN. As per the standard practice followed, all 19 patients were given a single injection of intravenous zoledronic acid (5 mg). All patients received oral daily supplements of calcium (500 $\mathrm{mg}$ ) and vitamin D (400 IU). Analgesics were given as and when required. Weight-bearing, as tolerated, was advised to all the patients. The patients were assessed at 2,6 , and 12 weeks after initiating the treatment and 6 monthly thereafter.

The collected data included demographic details of the patients and the visual analogue scale (VAS) pain score noted at the baseline visit. Gait and range of motion were assessed clinically, and anteroposterior and lateral radiographs of pelvis and MRI of both the hips were used for radiological evaluation. Clinical assessments and VAS pain scores were repeated at 2, 4, and 6 weeks follow-up visits. At week 12, patients underwent both clinical and radiological assessments along with an analysis of VAS pain score. The study involved radiological evaluation by two independent reviewers based on the comparison of baseline and 12th week MRI films. Subsequent follow-up at 6 monthly intervals included clinical evaluation, VAS score measurement, and analysis of radiographs of the hip.

Normality assumption was done for the data using Shapiro-Wilk test and the data was found to be normally distributed. VAS was analyzed from baseline to each followup visit using paired t-test and a p-value less than 0.05 
was considered as statistically significant. For each patient, the time of onset of symptoms and time required to become free from pain after initiating the treatment was also analyzed. Any adverse effects of the oral alendronate administration were also evaluated.

\section{RESULTS}

Mean age of the 19 patients was 42.1 years (range, 27-81 years) with 17 of them being males. Fifteen of them had acute onset of symptoms and rest had insidious onset of symptoms. Baseline MRI images of all the patients showed BME and none of them had AVN risk factors or any abnormalities in X-ray. Two patients neither had limp in their gait nor pain during a clinical assessment at the baseline visit. All patients had unilateral involvement (7-left hip and 12-right hip). The mean time of onset of symptoms was 4 weeks (range, 2-8 weeks). At baseline, 9 patients used a stick, 2 used walker, and 8 did not use any walking aid.

\section{Radiological assessment}

At 12-week follow-up visit, MRI analysis revealed complete resolution of baseline edema of the hip joint in 16 out of 19 patients $(84.21 \%)$ (Table 1$)$. Three patients with edema had lesser BME as compared to a baseline which resolved completely at 6 months as revealed by MRI. At a mean follow-up of 35 months, none of the patient's radiographs showed changes suggestive of AVN.

\section{MRI figures}

The pretreatment coronal T2-weighted MRI images

Table 1. Radiological and clinical assessment

\begin{tabular}{lcc}
\hline \multicolumn{1}{c}{ Efficacy parameter } & Baseline & 12 weeks \\
\hline Radiological assessment & & \\
MRI & & \\
\hline Edema present & $19(100)$ & $3(15.79)$ \\
\hline Edema absent & $0(0)$ & $16(84.21)$ \\
\hline X-ray & & \\
$\quad$ Normal & $19(100)$ & $19(100)$ \\
\hline Abnormal & $0(0)$ & $0(0)$ \\
Clinical assessment & & \\
Gait & $15(78.95)$ & $0(0)$ \\
\hline Limp & $4(21.05)$ & $19(100)$ \\
\hline No limp & & \\
Range of motion & $17(89.47)$ & $0(0)$ \\
\hline Painful & $2(10.53)$ & $19(100)$ \\
\hline Painless &
\end{tabular}

Values are presented as number (\%).

MRI, magnetic resonance imaging.
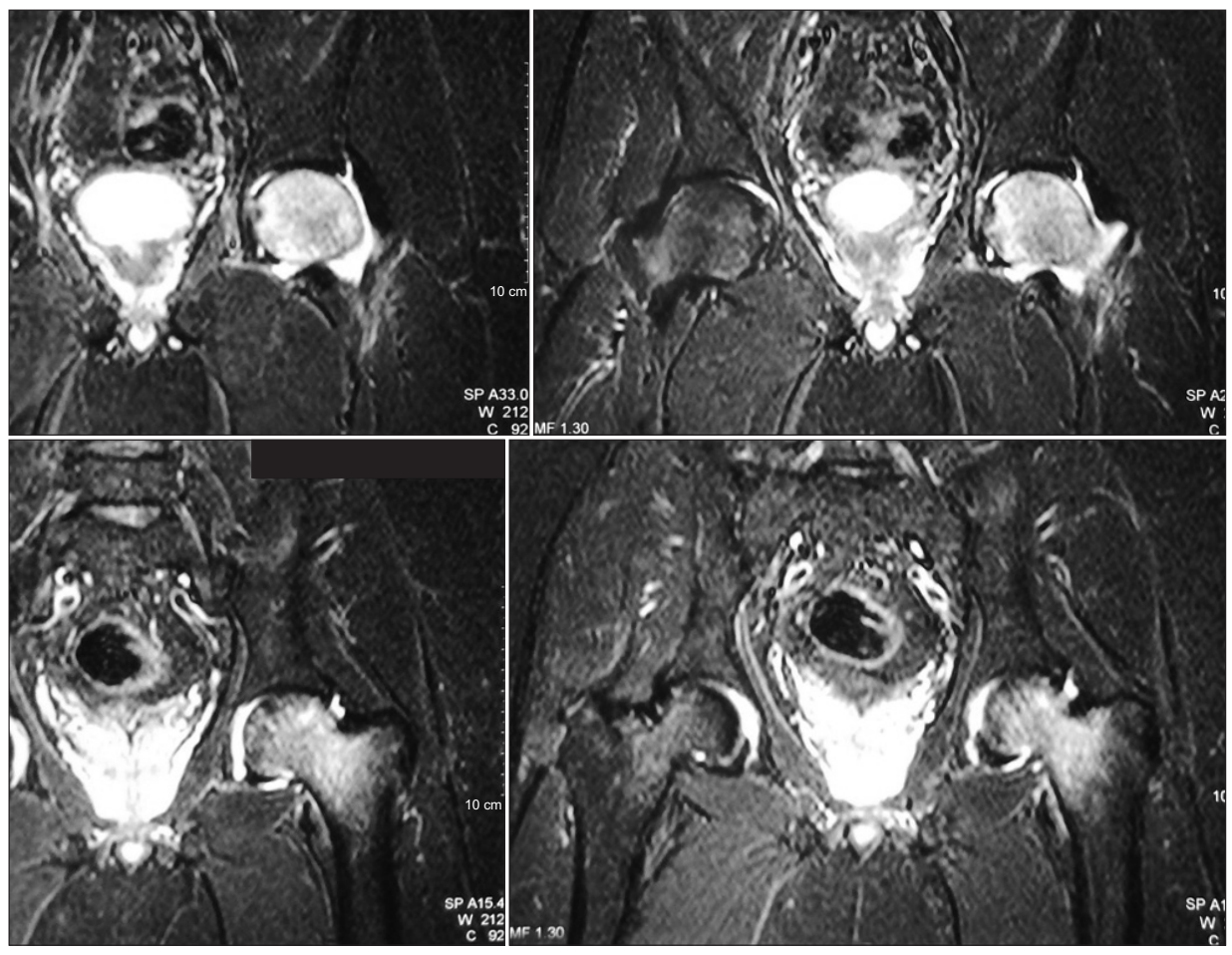

Fig. 1. Pre-treatment magnetic resonance imaging pictures of a 44-year-old patient suffering from transient osteoporosis of the left hip. 
(Fig. 1) shows diffused high signal intensity of the right femoral head, neck, and intertrochanteric region indicative of BME. The diffuse edema pattern is extended from the superior articular surface of the femoral head to the femoral neck and intertrochanteric region. The images reveal the absence of any focal lesion, thus ruling out the presence of AVN. This finding established the diagnosis of TOH in the patients. The post-treatment MRI images (coronal T2-weighted MRI) (Fig. 2) taken after 3 months of treatment with zoledronic acid shows complete resolution of BME in $84.2 \%$ of cases. Fig. 3 shows the pre- and post-treatment MRI pictures of another patient who was able to sit cross-legged after the therapy.

\section{Clinical assessment}

On clinical examination at week 12 , none of the patients had either limp or pain during hip joint movement (Table 1). VAS pain scores reduced consistently with each follow-up visit as compared to baseline and was statistically significant $(\mathrm{p}<0.0001)$ (Table 2$)$. The mean time required by the patients to get pain free after initiating the zoledronic acid treatment was 2.8 weeks (range, 2-5 weeks). No adverse events or adverse drug reactions were reported during the study period. At a mean follow-up of 35 months (range, 6-54 months), all the patients were pain-free and were able to continue with their normal routine activities without any limitations. None of the
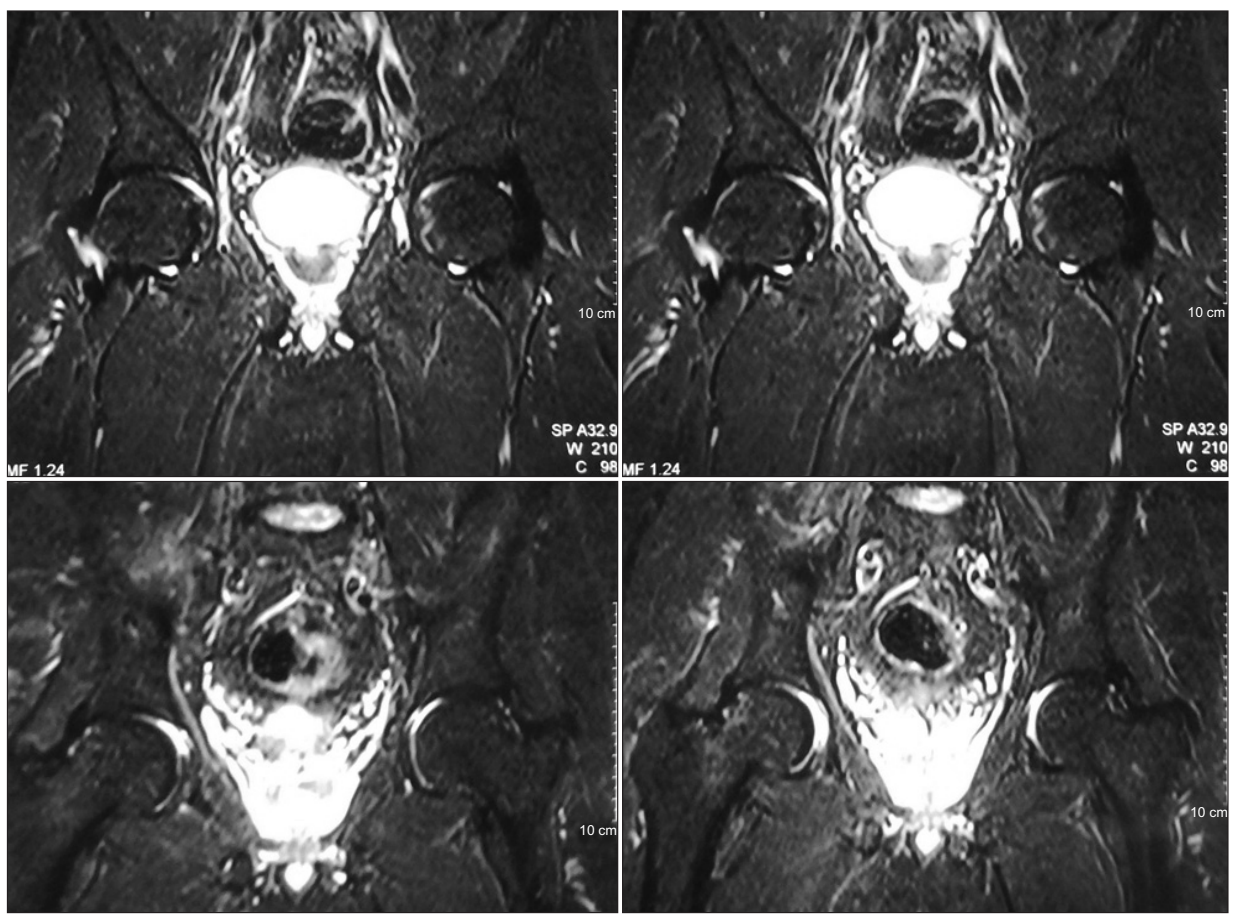

Fig. 2. Post-treatment magnetic resonance imaging pictures of the same patient at 4 months after treatment with zoledronic acid showing complete resolution of marrow edema.
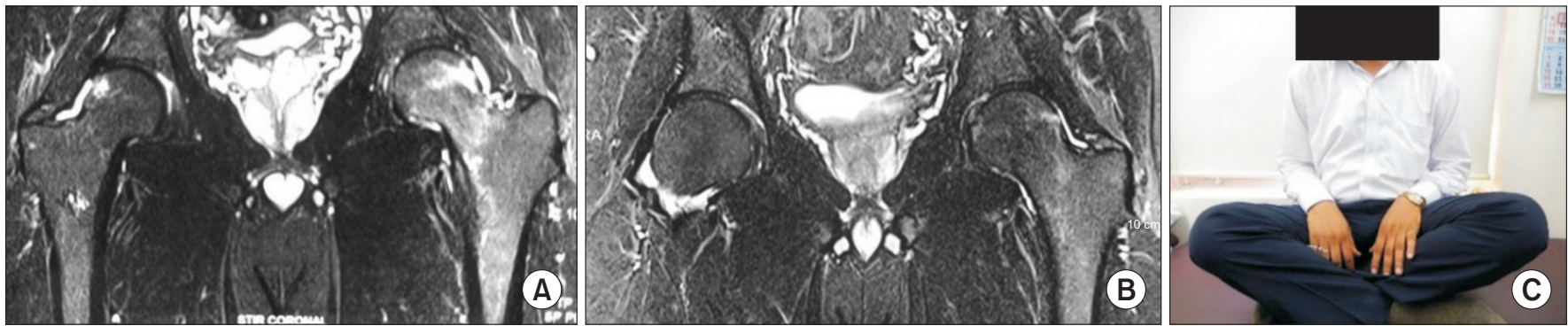

Fig. 3. (A) Pre-treatment magnetic resonance imaging (MRI) pictures of a 35-year-old patient suffering from transient osteoporosis of left hip. (B) Post-treatment MRI pictures at 3 months after treatment with zoledronic acid showing complete resolution of marrow edema. (C) Clinical picture showing good functional outcome at 4 months after treatment with zoledronic acid for transient osteoporosis of hip. 
Table 2. Visual analogue scale pain score at all follow-up visits

\begin{tabular}{lcccc}
\hline & Baseline & 2 weeks & 4 weeks & 6 weeks \\
\hline Visual analogue scale & $7.16(5-9)$ & $2.11(0-4)$ & $0.74(0-3)$ & $0.00(0-0)$ \\
p-value (t-test) & & $<0.0001$ & $<0.0001$ & $<0.0001$ \\
\hline
\end{tabular}

Values are presented as mean (range).

cases showed progression to AVN of the hip, at a mean follow-up of 35 months.

There were no major side effects noted necessitating cessations of the therapy in any of the patients. At a mean follow-up of 35 months, none of the patients reported any other side effect of the therapy.

\section{DISCUSSION}

$\mathrm{TOH}$ is a self-limiting condition which can be managed conservatively and resolves within 6-12 months; however, patients have to suffer from pain and limited mobility which adversely affects their quality of life throughout the time period required for resolution. Although exact etiopathogenesis of $\mathrm{TOH}$ remains unknown, various theories have been proposed including regional acceleratory phenomenon (RAP), microvascular injury as well as metabolic and endocrine factors [13,14]. A retrospective study of 10 male patients with $\mathrm{TOH}$ managed conservatively showed average time of 7.5 months (range, 4-11 months) for complete BME resolution on MRI and an average time of 5.8 months (range, 2-10 months) for complete resolution of clinical symptoms [2]. Apparently, various treatments have been tried to shorten the course of the disease. Among the various pharmacological therapies, treatment with bisphosphonates has shown promising results $[3,6,7]$.

Although the mechanism of action of bisphosphonates in $\mathrm{TOH}$ is still not proven, theories suggest that reduction in pain and resolution of disease are likely attributable to the drug's anti-inflammatory properties and inhibitory effects on osteoclasts $[9,15]$. McCarthy [16] revealed the presence of osteoclastic bone resorption in 14 out of 19 retrieved histological biopsy specimens from patients with $\mathrm{TOH}$. Further, all specimens showed thinning of trabeculae, a finding consistent with radiographic osteopenia along with the presence of edema and lymphocytes [16]. This histological study clearly demonstrates two pathogenesis involved in $\mathrm{TOH}$, increased osteoclastic activity and bone resorption, and the presence of edema.
Consequently, bisphosphonates were proposed as beneficial agents in reversing the disease process due to their anti-inflammatory and anti-osteoclastic activities.

Studies have shown that although alendronate is effective in completely reversing the disease process, its effect is observed late and takes from 6 weeks to 6 months for the complete resolution of symptoms $[3,6,8]$. In a study by Kibbi et al. [3], in two TOH patients treated with oral alendronate (70 mg/week), complete resolution of the symptoms was observed at 6 weeks in one patient and at 2 months in another patient. In another recent retrospective analysis of 14 hips treated conservatively using oral bisphosphonates, the mean period for resolution of symptoms was 17.1 weeks (range, 13-25 weeks) [8]. Until date, only one study has evaluated MRI changes along with clinical outcomes in 8 patients with TOH treated with alendronate $(70 \mathrm{mg} /$ week) for 6 months and demonstrated complete resolution of BME and improvement in clinical assessment at 6 months [6].

Intravenous pamidronate administered every month for 3 months led to resolution of symptoms in 2 months [9]. Similarly, intravenous ibandronate administered at a single dose of $3 \mathrm{mg}$ and an optional second dose of 2 $\mathrm{mg}$ in 12 cases has shown complete pain relief within a month [10]. Although other intravenous bisphosphonates have shown promising results, studies have shown that intravenous zoledronic acid is better than pamidronate and other intravenous bisphosphonates with regards to efficacy and patient tolerability [17]. Consequently, in our study, we have used a single dose of intravenous zoledronic acid because of the shorter infusion time and higher potency compared to other intravenous bisphosphonates [17].

In historical control, where no treatment was offered to the patients diagnosed with $\mathrm{TOH}$, an average time of 7.5 months (range, 4-11 months) was required for complete BME resolution on MRI and an average time of 5.8 months (range, 2-10 months) for resolution of clinical symptoms [2]. Herein, it was observed that patients diagnosed with $\mathrm{TOH}$ and treated with zoledronic acid exhib- 
ited significant clinical and radiological improvements as compared to the baseline. All patients were pain-free within an average of 2.8 weeks and had no limp in their gait. The pre-treatment and post-treatment MRI clearly showed resolution of BME (on week 12) in approximately $84 \%$ of patients $(16 / 19)$ after 3 months of treatment. There is still a controversy about whether $\mathrm{TOH}$ progresses to AVN or is a distinct entity with no future progression to AVN. Turner et al. [18] demonstrated the development of diffuse pattern seen in $\mathrm{TOH}$ into the focal pattern seen in AVN in six patients. A few authors reported that the focal necrosis seen in histological samples of TOH cases is consistent with stage 1 or 2 of AVN [19]. On the contrary, Yamamoto et al. [20] reported the absence of osteonecrotic lesions in the histopathological samples of TOH cases. Similarly, Holzer et al. [21] demonstrated that 48 patients (54 hips) when left untreated showed no progression to AVN at a mean follow-up of 43 months (range, 12-106 months). Consequently, reported literature has shown no convincing evidence regarding the progression of $\mathrm{TOH}$ to AVN. Although, Emad et al. [6] believe that most of the patients with TOH may not be diagnosed early and when they seek medical attention, many of them would have already progressed to AVN. It is proposed that patients diagnosed with TOH should be treated with bisphosphonates as a large number of studies have demonstrated the promising role of bisphosphonates in preventing collapse and progression of the disease to AVN $[22,23]$.

This study is a case series and has limitations which include lack of a randomized, double-blind prospective study design, and lack of a comparison group. Although, when compared with historical control, the presented therapy of a single dose of intravenous zoledronic acid provided earlier relief in pain and shortened the natural history of TOH. Secondly, based on the rarity of the disease, the literature until date comprises mostly of published case reports.

In conclusion, this study provides an effective pharmacotherapy option for patients with TOH. Our experience with an intravenous single dose of zoledronic acid reveals shortening of the natural course of the disease, reflected in terms of pain relief as early as 2 weeks and normalization of MRI changes at 3 months. Furthermore, all the cases were observed to be performing well at their last follow-up (mean, 35 months) and none had progressed to AVN. We thus present an effective pharmacotherapy in the management of $\mathrm{TOH}$.

\section{CONFLICT OF INTEREST}

No potential conflict of interest relevant to this article was reported.

\section{AUTHOR CONTRIBUTION}

Conceptualization: Agarwala S, Vijayvargiya M. Methodology: Agarwala S, Vijayvargiya M. Formal analysis: Agarwala S, Vijayvargiya M. Funding acquisition: none, Project administration: Agarwala S, Vijayvargiya M. Visualization: Vijayvargiya M. Writing - original draft: Agarwala S, Vijayvargiya M. Writing - review and editing: Agarwala S, Vijayvargiya M. Approval of final manuscript: all authors.

\section{REFERENCES}

1. Lakhanpal S, Ginsburg WW, Luthra HS, Hunder GG. Transient regional osteoporosis. a study of 56 cases and review of the literature. Ann Intern Med 1987;106:44450.

2. Balakrishnan A, Schemitsch EH, Pearce D, McKee MD. Distinguishing transient osteoporosis of the hip from avascular necrosis. Can J Surg 2003;46:187-92.

3. Kibbi L, Touma Z, Khoury N, Arayssi T. Oral bisphosphonates in treatment of transient osteoporosis. Clin Rheumatol 2008;27:529-32.

4. Arayssi TK, Tawbi HA, Usta IM, Hourani MH. Calcitonin in the treatment of transient osteoporosis of the hip. Semin Arthritis Rheum 2003;32:388-97.

5. Meizer R, Meraner D, Meizer E, Radda C, Landsiedl F, Aigner N. Outcome of painful bone marrow edema of the femoral head following treatment with parenteral iloprost. Indian J Orthop 2009;43:36-9.

6. Emad Y, Ragab Y, El-Shaarawy N, Rasker JJ. Transient osteoporosis of the hip, complete resolution after treatment with alendronate as observed by MRI description of eight cases and review of the literature. Clin Rheumatol 2012;31:1641-7.

7. Seok H, Kim YT, Kim SH, Cha JG. Treatment of transient osteoporosis of the hip with intravenous zoledronate: a case report. Ann Rehabil Med 2011;35:4325. 
8. Vaishya R, Agarwal AK, Kumar V, Vijay V, Vaish A. Transient osteoporosis of the hip: a mysterious cause of hip pain in adults. Indian J Orthop 2017;51:455-60.

9. Varenna M, Zucchi F, Binelli L, Failoni S, Gallazzi M, Sinigaglia L. Intravenous pamidronate in the treatment of transient osteoporosis of the hip. Bone 2002;31:96101.

10. Ringe JD, Dorst A, Faber H. Effective and rapid treatment of painful localized transient osteoporosis (bone marrow edema) with intravenous ibandronate. Osteoporos Int 2005;16:2063-8.

11. Waalen J. Current and emerging therapies for the treatment of osteoporosis. J Exp Pharmacol 2010; 2:121-34.

12. Saag K, Lindsay R, Kriegman A, Beamer E, Zhou W. A single zoledronic acid infusion reduces bone resorption markers more rapidly than weekly oral alendronate in postmenopausal women with low bone mineral density. Bone 2007;40:1238-43.

13. Trevisan C, Ortolani S, Monteleone M, Marinoni EC. Regional migratory osteoporosis: a pathogenetic hypothesis based on three cases and a review of the literature. Clin Rheumatol 2002;21:418-25.

14. Harrington S, Smith J, Thompson J, Laskowski E. Idiopathic transient osteoporosis: a hidden cause of hip pain. Phys Sportsmed 2000;28:82-96.

15. Schapira D, Braun Moscovici Y, Gutierrez G, Nahir AM. Severe transient osteoporosis of the hip during pregnancy. Successful treatment with intravenous biphosphonates. Clin Exp Rheumatol 2003;21:107-10.
16. McCarthy EF. The pathology of transient regional osteoporosis. Iowa Orthop J 1998;18:35-42.

17. Zhao X, Hu X. Dosing of zoledronic acid with its antitumor effects in breast cancer. J Bone Oncol 2015;4:98101.

18. Turner DA, Templeton AC, Selzer PM, Rosenberg AG, Petasnick JP. Femoral capital osteonecrosis: MR finding of diffuse marrow abnormalities without focal lesions. Radiology 1989;171:135-40.

19. Plenk H Jr, Hofmann S, Eschberger J, Gstettner M, Kramer J, Schneider W, et al. Histomorphology and bone morphometry of the bone marrow edema syndrome of the hip. Clin Orthop Relat Res 1997;(334):7384.

20. Yamamoto T, Kubo T, Hirasawa Y, Noguchi Y, Iwamoto Y, Sueishi K. A clinicopathologic study of transient osteoporosis of the hip. Skeletal Radiol 1999;28:621-7.

21. Holzer I, Snir N, Ben-Galim P, Maman E, Rosenblatt Y, Dekel S. Transient osteoporosis of the hip: long-term outcomes in men and nonpregnant women. Curr Orthop Pract 2009;20(2):161-3.

22. Agarwala S, Shah S, Joshi VR. The use of alendronate in the treatment of avascular necrosis of the femoral head: follow-up to eight years. J Bone Joint Surg Br 2009; 91:1013-8.

23. Lai KA, Shen WJ, Yang CY, Shao CJ, Hsu JT, Lin RM. The use of alendronate to prevent early collapse of the femoral head in patients with nontraumatic osteonecrosis: a randomized clinical study. J Bone Joint Surg Am 2005;87:2155-9. 